\title{
Die heterosexuelle Familie als Norm
}

\section{Das heteronormative Regime der rechtlichen Regulierung gleichgeschlechtlicher Elternschaft}

Der Wandel von Familie wird seit Jahrzehnten wissenschaftlich untersucht und gesellschaftlich diskutiert. Neuerdings geschieht dies auch hinsichtlich lesbisch, schwul, bisexuell oder transgender/trans* $\left(\mathrm{LGBT}^{*} \mathrm{Q}\right)$ lebenden Eltern. Sogenannte "Regenbogenfamilien" werden in Medien als neue Familien portraitiert und politisch wird darüber diskutiert, ob und wie queere Elternschaft rechtlich anerkannt werden soll. Insbesondere von rechtspopulistischer und christlich-konservativer Seite formiert sich jedoch verstärkt Gegenwehr gegen die zunehmende rechtliche Anerkennung von Familien mit gleichgeschlechtlichen Eltern. Wie lässt sich verstehen, dass Regenbogenfamilien rechtliche Anerkennung erfahren und zugleich die heterosexuelle Familie weiterhin als die Norm gilt? Welche Rolle spielen dabei Kinder- und Jugendschutzbehörden?

L GBT* Q-Personen $^{1}$ haben seit jeher Familie neu gefasst. Sie haben sich (oft gezwungenermaßen) von ihrer Herkunftsfamilie abgegrenzt und eine Auffassung von Familie entwickelt, die über leiblich oder genetisch miteinander verbundene Gemeinschaften hinausgehen. Seit Ende der 1960er Jahre und mit Erstarken der LGBT*Q-Emanzipationsbewegung wurde die (Wahl-) Familie als Ausdruck einer Zusammengehörigkeit unter LGBT*Q, die füreinander Sorge tragen, verwendet (Weston 1991; Newton 1993). (Lesbische) Feminist_innen - insbesondere Feminist_innen of $\mathrm{Co}$ lor - haben bereits früh die Institutionen der Ehe und Familie aufgrund ihrer patriarchalen, sexistischen, homonormativen und rassisierenden Privilegienverteilung

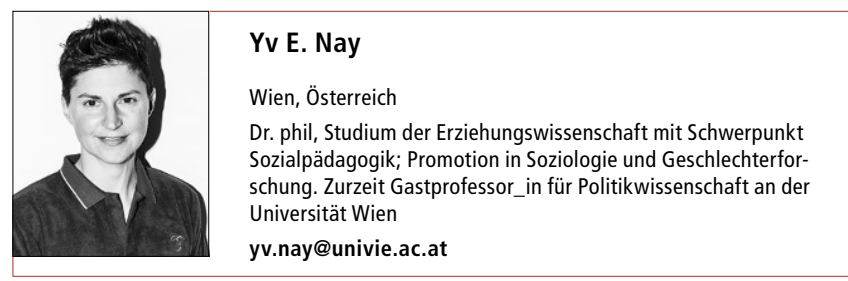

Zusammenfassung Dieser Beitrag thematisiert am Beispiel der Schweiz, wie im Rahmen der politischen Forderungen nach rechtlicher Anerkennung queerer Familien einerseits eine Erweiterung der rechtlichen Auffassung von Familie erfolgt, während gleichzeitig andererseits die heterosexuelle Familie als die Norm bestärkt wird. Er formuliert Überlegungen, was diese politischen Prozesse für die Arbeit von Kinder- und Jugendschutzbehörden bedeuten, die die rechtlichen Neuerungen rund um Regenbogenfamilien umsetzen müssen.

Schlüsselwörter Wandel von Familie, LGBT*Q, Queere Familien, Regenbogenfamilien, Kindeswohl, Heteronormativität radikal infrage gestellt. ${ }^{2}$ Sie verwenden seit den 1970er Jahren Verwandtschaftsbegriffe, wenn sie Mitaktivist_ innen als Schwestern bezeichnen (Piesche 2012; Women of Black Heritage 2003; Moraga und Anzaldúa 1983 [1981]; Kokula und Böhmer 1991).

Obwohl Familie bereits seit längerem kritisch betrachtet, umgedeutet und als Ausdruck für Solidarisierung gefasst wird, gelten heute vor allem LGBT* $\mathrm{Q}$ mit Kindern respektive so genannte Regenbogenfamilien als die „neuen “, „alternativen“ Familien (Funcke und Thorn 2010; Eggen und Rupp 2011). Diese verengte Auffassung zeigt sich exemplarisch in politischen Debatten in der Schweiz zur rechtlichen Regelung von Elternschaft von Elternpaaren des gleichen Geschlechts, die ich im Rahmen meiner Forschung untersucht habe (Nay 2017; siehe auch Mesquita 2011).

In diesem Beitrag präsentiere ich ausgewählte Ergebnisse meiner Studie zur Frage, wie queere Familien politisch debattiert und rechtlich reguliert werden. Ich zeige, wie im Rahmen der politischen Forderungen nach rechtlicher Anerkennung queerer Familien eine Erweiterung der rechtlichen Auffassung von Familie erfolgt, während gleichzeitig die heterosexuelle Familie als Norm bestärkt wird. Abschließend formuliere ich einige Überlegungen, was diese politischen Prozesse für die Arbeit von Behörden (beispielsweise die Kinder-, Jugend- und Erwachsenenschutzbehörden) bedeutet, die die rechtlichen Neuerungen rund um Regenbogenfamilien umsetzen müssen.

\section{Regenbogenfamilien als Politikum}

Die politischen Debatten rund um Regenbogenfamilien gründen auf dem Bundesgesetz zur eingetragenen 
Partnerschaft gleichgeschlechtlicher Paare (PartG), das 2007 in der Schweiz in Kraft trat (PartG 2007). Im Gegensatz zum Eherecht ist dieses Gesetz nicht Teil des Familienrechts des Schweizerischen Zivilgesetzbuches (ZGB) und verwehrt eingetragenen Paaren die Adoption sowie den Zugang zu fortpflanzungsmedizinischen Technologien (vgl. Art. 28 PartG 2007). ${ }^{3}$ Diese Regelungen werden von $\mathrm{LGBT}^{*} \mathrm{Q}$-Organisationen angefochten. Während die Öffnung der Ehe für gleichgeschlechtliche Paare weiterhin debattiert wird, haben die legislativen Instanzen der Schweiz einer rechtlichen Regelung für die Stiefkindadoption durch gleichgeschlechtliche Paare kürzlich zugestimmt (Art. 264 ZGB 2018). Die Adoption von Kindern, die keinerlei leiblich-genetischen Bezug zum adoptierenden gleichgeschlechtlichen Paar hat sowie der Zugang zu medizinisch assistierten Reproduktionstechnologien bleiben jedoch weiterhin verboten. Weitergehende Rechte der grundsätzlichen Ermöglichung und Absicherung familialer Nähe- und Fürsorgebeziehungen wie beispielsweise die Berücksichtigung von Kindsverhältnissen mit mehr als zwei Elternteilen wurden nicht diskutiert.

Dementsprechend kreist die politische Diskussion im Schweizer Bundesparlament durchgängig um das Thema der Adoption durch gleichgeschlechtliche Paare. Dabei wird die Maxime des Kindeswohls - d. h. die Frage, ob das Wohlergehen von Kindern, die mit einem Elternpaar des gleichen Geschlechts aufwachsen, gefährdet sei - in den politischen Debatten sowohl bei den Gegner_innen als auch bei den Befürworter_innen der Stiefkindadoption zum Dreh- und Angelpunkt für die rechtliche Anerkennung von Regenbogenfamilien. Aus der gegnerischen, rechtspopulistischen sowie christlich-konservativen Perspektive wird befürchtet, dass die Kinder aufgrund der Gleichgeschlechtlichkeit der Eltern einen Mangel an vorgelebten Geschlechtern erleiden. Dabei wird von einer dichotom angeordneten Zweiund Cis-Geschlechtlichkeit ${ }^{4}$ ausgegangen, die sich mittels einer heterosexuellen Beziehung zwischen zwei Personen ergänzt und die Grundlage für die menschliche Reproduktion bildet. Diese heteronormative Setzung wird als unabdingbare Voraussetzung für die Entwicklung einer eindeutigen Geschlechtsidentität gesetzt, die zudem in Einklang mit dem bei Geburt zugewiesenen Geschlecht sein soll. Daraus wird die Gefahr abgeleitet, dass Kinder mit gleichgeschlechtlichen Eltern kein heterosexuelles Begehren entwickeln können (Nay 2018). Auch gemäß den Befürworter_innen einer Öffnung der Stiefkindsadoption für gleichgeschlechtliche Paare gilt es, die befürchtete Gefährdung des Kindeswohls in gleichgeschlechtlichen Partner_innenschaften ernst zu nehmen. Sie teilen mit den Gegner_innen das Gefühl der
Befremdung durch die Vorstellung, ein Kind könne bei gleichgeschlechtlichen Eltern aufwachsen. Nichtsdestotrotz sprechen sie sich mehrheitlich für die Gewährung von Stiefkindadoptionsrechten für gleichgeschlechtliche Paare aus. Ausschlaggebend ist für diesen Entscheid die Tatsache, dass die Familienverhältnisse mit gleichgeschlechtlichen Eltern bereits bestehen und diese durch das Verwehren von Rechten folglich nicht verhindert werden können. Das Verbot der gemeinschaftlichen Adoption wird hingegen beibehalten (Nay 2019).

\section{Das Kindeswohl als Anker heteronormativer Familienvorstellungen}

Die eben skizzierten Positionen in den politischen Debatten um Anerkennung von „Regenbogenfamilien“ zeigen in dreierlei Hinsicht, wie ein bestimmter Wandel von Familie rechtlich anerkannt wird und dabei allerdings von heteronormativen Vorstellungen von Familie begleitet wird:

- Erstens zeigt die politische Diskussion, dass die rechtliche Anerkennung und die damit verbundene Erweiterung von Normalitätsvorstellungen von Familie deutliche Grenzen haben. Denn die Einbindung in die herrschende Auffassung von Familie können nur diejenigen erlangen, die sich an die hierfür notwendigen normativen Vorgaben anpassen können und wollen, d. h. Familien bestehend aus einem LGBT* Q-Liebesund Elternpaar mit Kind(ern). Dieser normalisierende Einschluss weitet die hegemoniale Grenze einer „normalen“ Familie von zwei- und cis-geschlechtlichen Paaren mit Kind(ern) auf gleichgeschlechtliche Paare mit Kind(ern) aus. Allerdings bleiben die bestehenden vielfältigen Konstellationen der Fürsorge von $\mathrm{LGBT}^{*} \mathrm{Q}$ rechtlich weiterhin unabgesichert. Die familiale Vielfalt, wie ich sie eingangs skizziert habe, erfährt durch diesen normalisierenden Einschluss eine Verengung (Nay 2017, S. 116-127).

- Zweitens zeigen die Debatten, dass die Einführung von Elternrechten für gleichgeschlechtliche Paare trotz der Erweiterung dessen, was rechtlich als Familie gilt, die Norm der heterosexuellen Familie bestätigt. Denn die Beschränkung auf Stiefkindadoptionsrechte bedeutet, dass gleichgeschlechtliche Elternpaare, die gemeinsam ein Kind planen und erziehen, im Gegensatz zu verschiedengeschlechtlichen Elternpaaren keine Elternrechte per Geburt des Kindes erhalten, sondern per se das Verfahren der Stiefkindadoption durchlaufen müssen. Originäre Elternschaft wird dadurch als heterosexuelle Elternschaft festgeschrieben. Zugleich werden gleichgeschlechtlichen Elternpaaren Stiefkindadoptionsrechte zugestanden, die allerdings auf Familien ausgerichtet, in denen ein Eltern- 
teil unbekannt, verstorben oder nicht Teil des Alltags des Kindes ist und infolgedessen als Elternteil durch einen sogenannten Stiefelternteil ersetzt wird. Diese Form der Elternrechte ist auf heterosexuelle Eltern ausgerichtet, die als eine sogenannte Patchworkfamilie leben. Der größte Teil queerer Familien hingegen plant, zeugt, gebärt und erzieht ein Kind oder mehrere Kinder gemeinsam; rechtlich aber werden sie wie Fortsetzungsfamilien mit heterosexuellen Eltern behandelt, in denen ein Stiefkindelternteil das Kind der Partnerin oder des Partners adoptiert. Damit bleibt zum einen die gelebte Situation gleichgeschlechtlicher Elternschaft unberücksichtigt, zum anderen wird die verschiedengeschlechtliche Elternschaft bei Geburt als Norm bestätigt.

- Drittens wird in den erwähnten politischen Debatten deutlich, dass die Ausgestaltung dessen, was unter Kindeswohl rechtlich zu fassen ist, nicht festgeschrieben ist und deshalb der Interpretation bedarf. In den Debatten um Adoptionsrechte für gleichgeschlechtliche Paare wird das Kindeswohl durchgängig anhand heteronormativer Vorstellungen definiert. Das Wohlergehen eines Kindes zeigt sich, folgen wir den Argumentationen in der Schweizer Bundesparlamentsdebatte, wenn das Kind sich nicht als trans*-, sondern als cis-gender identifiziert und sich in seinen Begehrensstrukturen dem Gegengeschlecht zuwendet. Was die Verfechter_innen einer derartigen Auffassung von Kindeswohl nicht bedenken (wollen) ist, dass gerade ein solch striktes und enges Verständnis kindlicher Entwicklung homo- und trans*-phobe gesellschaftliche Verhältnisse (re-)produziert, die das Wohlergehen nicht nur von Kindern aus Regenbogenfamilien, sondern von Kindern insgesamt gefährdet. Denn eine derartige Setzung dessen, was unter Kindeswohl verstanden wird, bietet keinerlei Schutz des Rechtes von Kindern auf die freie Entfaltung einer geschlechtlichen Identifikation und sexuellen Lebensweise.

\section{Folgerungen für die Arbeit von Kinder- und Jugendschutzbehörden}

Die Ausführungen zur Stiefkindadoption gleichgeschlechtlicher Elternpaare zeigen, dass derzeitige Gesellschaftsordnungen auf Veränderungen familialer Lebensweisen nicht alleine mit Ausschließung reagieren, sondern ebenso mit Prozessen einschließender Normalisierung und differenzierter, wenn auch hierarchischer sozialer Integration. Wir haben es also mit einer Verschiebung der Norm zu tun, die sich allerdings nicht auflöst. Vielmehr wird die Norm bestätigt, indem eine eingeschränkte rechtliche Regulierung auf „Andere“, nicht heterosexuelle Familien, ausgeweitet wird.
Für die Arbeit der zuständigen Kinder- und Jugendschutzbehörden, die neuerdings mit Stiefkindadoptionsverfahren in Familien mit gleichgeschlechtlichen Eltern konfrontiert sind, hat das Konsequenzen. Sie stehen vor neuartigen Familienformationen, die in ihrer bisherigen Arbeit nicht von Belang waren. Die hier skizzierten politischen Debatten verweisen auf die zentrale Bedeutung des Kindeswohls im Stiefkindsadoptionsverfahren. Das Kindeswohl ist der Dreh- und Angelpunkt für die Gewährung von Stiefkindsadoptionsrechten und damit auch der Ansatzpunkt für die Kinder- und Jugendschutzbehörden, um ein verantwortungsvolles Vorgehen in Stiefkindsadoptionsverfahren zu entwickeln. Dies betrifft hauptsächlich zwei Bereiche des Verfahrens:

- Die Beurteilung des Kindeswohls und

- die damit verbundene Anhörung des Kindes.

Aufgrund der politischen Debatten um das Kindeswohl ist es angezeigt, dass die für das Stiefkindsadoptionsverfahren zuständigen Behörden reflektiert mit heteronormativen Annahmen der kindlichen Entwicklung umgehen sollten. Dies beinhaltet nicht alleine, dass sie möglichen Befürchtungen, ein Kind mit gleichgeschlechtlichen Eltern könnte sich nicht nach heteronormativen Vorstellungen entwickeln, Einhalt gebieten. Darüber hinaus sollten Kinder- und Jugendschutzbehörden bei der Beurteilung des Kindeswohls in einem Verfahren der Stiefkindadoption berücksichtigen, dass es sich zumeist um eine Familie mit einem gleichgeschlechtlichen Elternpaar handelt, das gemeinsam Familie geplant und realisiert hat. Es geht in solchen Konstellationen folglich nicht darum, dass ein Elternteil ersetzt werden soll, sondern um die rechtliche Anerkennung bestehender Familienverhältnisse.

Diese Tatsache ist insbesondere für die Praxis der Anhörung der Kinder im Stiefkindsadoptionsverfahren zentral. Was in Fortsetzungsfamilien durchaus Sinn machen kann, ist für von Anbeginn an geplante Familien, wie es gleichgeschlechtliche Eltern mit Kindern oft sind, nicht dem eigentlichen Zweck entsprechend. In einem Stiefkindsadoptionsverfahren werden Kinder in der Regel ab dem sechsten Lebensjahr angehört und ab einem urteilsfähigen Alter von ungefähr 14 Jahren soll das Kind der Stiefkindadoption zustimmen können (Büchler et al. 2018). Zumal bei einer geplanten Familie mit gleichgeschlechtlichen Eltern keinem dritten Elternteil Rechte genommen werden, sondern dem nicht gebärenden oder nicht zeugenden Elternteil Rechte zugestanden werden sollen, ist die Anhörung obsolet. In dieser Konstellation ist die Stiefkindadoption mit kei- 
nerlei Veränderungen im Alltag des Kindes und im $\mathrm{Zu}$ sammenleben mit seinen Eltern verbunden.

Wenn also das Familienleben nicht reorganisiert wird, die Stiefkindadoption lediglich die mit der Geburt entstandenen Verhältnisse rechtlich bestätigen soll, kein bestehendes Kindesverhältnis durch die Stiefkindadoption hinfällig wird, dann ist die Anhörung des Kindes nicht zweckmäßig (siehe auch Büchler et al. 2018). Vielmehr wird in solchen Familienkonstellationen die Anhörung zum Ausdruck des Misstrauens gegenüber gleichgeschlechtlichen Eltern und damit zu einer diskriminierenden Intervention.

Aus dem hier präsentierten Beispiel der rechtlichen Regelung gleichgeschlechtlicher Elternschaft in der Schweiz lässt sich folgern, dass politische Instanzen und ausführende Behörden von hetero- und cis-normativen Annahmen dessen, was Familie ist und wie kindliche Entwicklung verlaufen soll, geprägt sind. Eine tatsächliche rechtliche und soziale Anerkennung bedarf einer kritischen Reflexion dieser Annahmen durch die Beteiligten. Erst dann kann deutlich werden, dass die bestehenden Regelungen weit davon entfernt sind, die tatsächlich gelebten, vielfältigen Familienverhältnisse rechtlich anzuerkennen und abzusichern.

Dieser Beitrag basiert auf meiner Studie zu familialen Lebensweisen von LGBT*Q-Personen und deren gesellschaftspolitischer Regulierung (Nay 2017), die vom Schweizerischen Nationalfonds und von der Universität Basel gefördert wurde.

Funding. Open access funding provided by University of Vienna.

Open Access. Dieser Artikel wird unter der Creative Commons Namensnennung 4.0 International Lizenz (http://creativecommons.org/ licenses/by/4.0/deed.de) veröffentlicht, welche die Nutzung, Vervielfältigung, Bearbeitung, Verbreitung und Wiedergabe in jeglichem Medium und Format erlaubt, sofern Sie den/die ursprünglichen Autor(en) und die Quelle ordnungsgemäß nennen, einen Link zur Creative Commons Lizenz beifügen und angeben, ob Änderungen vorgenommen wurden.

1. Trans"-Personen ist eine Bezeichnung für Menschen, die sich nicht mit dem ihnen bei Geburt zugewiesenen Geschlecht identifizieren. Der Asterisk fungiert als Platzhalter für verschiedene Begriffe und Bedeutungen von Transgeschlechtlichkeit.

2. In ähnlicher Wiese kritisieren heute queer-theoretische Arbeiten die politische Agenda von gewissen LGBT*Q-Organisationen für ibre politischen Bestrebungen nach Einschluss in neoliberale, nationalistische, rassistische, kapitalistische Rechtsordnungen, wie beispielsweise die Ehe für alle (vgl. beispielsweise Duggan 2003; Eng et al. 2005).

3. Trotz dieser Verbote werden $L G B T^{*} Q$ als Eltern im Part $G$ erwähnt: Im Falle einer Trennung eingetragener Partner_innen mit Kindern sieht das Gesetz eine Unterstützungspflicht (Art. 27 Abs. 1
PartG) vor und räumt dem nicht-biologischen Elternteil Besuchsrechte ein (Art. 27 Abs. 2 PartG).

4. Das Präfix „Cis“ bezeichnet die Geschlechtlichkeit von Menschen, die im ihnen bei Geburt zugeordneten Geschlecht leben und sich damit identifizieren.

\section{Literatur}

Büchler, A., Cottier, M., Jaffé, P. D., \& Simoni, H. (2018). Empfehlungen zur Anhörung des Kindes im Verfahren der Adoption durch gleichgeschlechtliche Paare. 1-10. https://www.unige.ch/cide/files/3315/2904/4857/ Anhoerung_Kinder_Adoption_Regenbogenfamilien.4.6.18.pdf. Zugegriffen: 20. Aug. 2019.

Bundesgesetz über die eingetragene Partnerschaft gleichgeschlechtlicher Paare (PartG) vom 18. Juni 2004, in Kraft per 1. Januar 2007.

Duggan, L. (2003). The twilight of equality? Neoliberalism, cultural politics, and the attack on democracy. Boston: Beacon Press.

Eggen, B., \& Rupp, M. (2011). Gleichgeschlechtliche Paare und ihre Kinder. Hintergrundinformationen zur Entwicklung gleichgeschlechtlicher Lebensformen in Deutschland. Zeitschrift für Familienforschung, 11(7), 23-37.

Eng, D., Halberstam, J., \& Muñoz, J. E. (2005). What's queer about queer studies now? Social Text, 3-4, 1-17.

Funcke, D., \& Thorn, P. (Hrsg.). (2010). Die gleichgeschlechtliche Familie mit Kindern. Interdisziplinäre Beiträge zu einer neuen Lebensform. Bielefeld: transcript.

Kokula, I., \& Böhmer, U. (1991). Die Welt gehört uns doch! Zusammenschluss lesbischer Franen in der Schweiz der 30er Jahre. Zürich: eFeF.

Mesquita, S. (2011). Ban Marriage! Ambivalenzen der Normalisierung aus queer-feministischer Perspektive. Wien: Zaglossus.

Moraga, C., \& Anzaldúa, G. (Hrsg.). (1983). This bridge called My back. Writings by radical women of color. New York: Kitchen Table Women of Color Press.

Nay, Y. E. (2017). Feeling Family. Affektive Paradoxien der Normalisierung von „Regenbogenfamilien". Wien: Zaglossus.

Nay, Y. E. (2018). Das „Kindeswohl“ im Rahmen von „Regenbogenfamilien-Politiken“. Zeitschrift psychosozial, 18(1), 79-88.

Nay, Y. E. (2019). Homonormative und nationalistische Politiken des Fortschritts in Debatten um nicht-hegemoniale Familien und Verwandtschaft. Gender. Journal for Gender, Culture and Society, 11(2), 41-55.

Newton, E. (1993). Cherry grove, fire Island. Sixty years in america's first gay and lesbian town. Boston: Beacon Press.

Piesche, P. (Hrsg.). (2012). „Euer Schweigen schützt Euch nicht“. Audre Lorde und die Schwarze Frauenbewegung in Deutschland. Berlin: Orlanda Frauenverlag.

Schweizerisches Zivilgesetzbuch ZGB (Adoptionsrecht), Änderung vom 17. Juni 2016, in Kraft per 1. Januar 2018.

Weston, K. (1991). Families we choose. Lesbians, gay men and kinship. New York: Columbia University Press.

Women of Black Heritage (2003). draussen - drinnen - dazwischen. Olympe. Feministische Arbeitshefte zur Politik (Sonderheft 18). Zürich: Olympe. 